\title{
The Description of Oil Displacement Mechanism in Steam Injection of Multi-Field Synergy with Exergy Transfer*
}

\author{
Qinglin Cheng, Yang Wang, Xiaoli Sun \\ Key Lab of Ministry of Education for Enhancing the Oil and Gas Recovery Ratio, \\ Northeast Petroleum University, Daqing, China \\ Email: chengqinglin7212@163.com
}

Received April 2, 2013; revised April 29, 2013; accepted May 7, 2013

Copyright (c) 2013 Qinglin Cheng et al. This is an open access article distributed under the Creative Commons Attribution License, which permits unrestricted use, distribution, and reproduction in any medium, provided the original work is properly cited.

\begin{abstract}
Steam injection is a most effective way for improving heavy oil recovery efficiency, and it has academic and practical significance for the mechanism of multi-field synergy oil displacement. Mechanism of "diversified" oil displacement which is obtained by traditional study methods in the exploitation territory of oil and gas fields has both respective roles and mutual cross shortages. To describe and analyze the displacement process of multi-field coupling with exergy transfer can simplify this kind of problem by introducing a unified goal-driving exergy. It needs to use the method of theoretical modeling, numerical simulation and experimental validation to study the basic law of exergy transfer in the oil displacement process of multi-field synergy, make a thorough research for the flooding process of steam injection with exergy transfer theory and reveal the oil displacement mechanism in steam injection of multi-field synergy. Thus the theory instruction and technical support can be provided to improve reservoirs producing degree and extraction ratio.
\end{abstract}

Keywords: Steam Injection; Exergy; Exergy Transfer; Multi-Field Synergy; Mechanism of Oil Displacement

\section{Introduction}

There are two ways of oil recovery in steam injection, first, steam puff and huff are proceeded, that is a large amount of steam is injected with the higher speed in the same well, the well is closed and stewed, and oil recovery is started when opening the well after several days. After many repeated cycles of steam puff and huff, in order to enhance the recovery efficiency, injection-production well pattern are composed of their groups as same as water driving, steam injection wells and production wells can be arranged by line well pattern or areal well pattern [1]. Steam injection is the most widely used method of heavy oil extraction in the world at present, and the remarkable effect for increasing the recovery efficiency is obtained. However, because of involving interactions of many complicated factors, there are many problems of steam injection recovery which should be resolved, no matter theoretical analysis or the injection process. To study deeply the oil displacement mechanism

*Foundation item: Supported by the National Natural Science Foundation of China (51106020, 51051002), Specialized Research Fund for the Doctoral Program of Higher Education (20102322110002) and Youth Scholar Backbone Supporting Plan Project of Heilongjiang General Colleges and Universities (1252G005). in multi-field synergy process is very important, since it can provide theoretical basis and technical support to improve the producing degree reservoirs and extraction efficiency [2].

\section{Traditional Method for Studying Mechanism of Oil Displacement}

In accordance with the fact that the production technique with steam injection is adopted to enhance the recovery efficiency in heavy oil reservoir, the comprehensive studies are developed in oil-gas field development domain in oil displacement mechanism, and more systematic research methods are formed, including numerical simulation and physical simulation. Numerical simulation and physical simulation, which depend on each other and have respective characteristics, are both main means of program designed for heavy oil thermal recovery and the verification basis of new method and technology of thermal recovery. All applied effects depend on the accuracy and complementary skills of "dual mode" [1].

\subsection{Numerical Simulation Technology}

According to the practical condition of oil reservoir, the 
numerical simulation study of heavy oil thermal recovery firstly should build its mathematical model which includes the partial differential equation describing the flow law of fluid in oil layer, state equation and definite condition describing the physicochemical properties of fluid. Then the basic physical parameters of numerical simulation in the oil reservoir are confirmed through history matching of reservoir object development. On the basis of the numerical model confirmed by history matching, the sensitivity of development mode is analyzed, development mode chosen and development effect optimized. Finally through the simulated prediction for the development effect, the predicted result of reservoir development is attained and its benefit is determined with economic evaluation followed by. Concretely speaking, the influence on development effect is analyzed due to the factors including steam injection rate, steam injection temperature, mass dryness fraction of steam injection, gas extraction mode, injection and production well pattern, etc. At the premise of enhancing the cumulative oil production, recovery percentage and cumulative oilgas ratio but reducing water-oil ratio and cumulative gas injection volume, the optimal injection-production parameters are optimized, which offers a theoretical basis for efficient development in oil field $[1,3]$.

\subsection{Physical Simulation Method}

Under the conditions of steam injection, numerical simulation is also difficult to describe technical process, reflect interaction between rock and fluid, and the other aspects. Many complicated physical and chemical changes in process of oil production are observed directly and necessary parameters for mathematical model are offered by physical simulation. Thus physical simulation becomes the important basis of numerical simulation, for instance, to determine oil-water relative permeability curve under different temperature conditions, to study the change law of oil-water relative permeability and the effect law of irreducible water saturation and residual oil saturation by heating, to experimentally compare the influence of temperature on efficiency of water flooding and steam flooding, to experimentally simulate the results of the recovery efficiency in various stages of turning steam driving under different moisture content times and the total recovery efficiency, to experimentally simulate recovery efficiency in different levels and residual oil saturation of steam flooding after water flooding, to analyze and the impact of steam overlay on sweep efficiency, to optimize the steam parameters, to develop the pilot test and so on $[1,4]$.

A large number of laboratory simulation experiments and numerical simulations indicate that mechanism of diversified oil displacement with steam injection can be united as seepage characteristics of heavy oil in porous media. For instance, rheology property of heavy oil: it shows character of pseudo plastic fluid at low temperature, but shows character of Newtonian fluid when heated to a certain temperature. The lager oil-water viscosity ratio, the more likely it is to cause fingering in macroscopic oil-water motion, so the effect of viscous oil recovery can be improved by reducing the oil viscosity and oil-water viscosity ratio. The oil displacement efficiency is influenced obviously by rock wettability, and high temperature steam can improve obviously the oil displacement efficiency by changing the rock-wet into water-wet. The relative permeability curve drawn in the laboratory is the comprehensive embodiment of the effect of crude oil displacement in cores, reflecting the functions of many parameters including pore structure, wettability, viscosity ratio of displacing fluid and driven fluid, thermal expansion, thermal distillation, etc. [1].

Although the oil displacement mechanism can be summarized completely with seepage flow character of heavy oil in porous media as the core, it has both respective roles and shortages of mutual cross. It is necessary to analyze the synergy mechanism of "diversified" mechanism from a new point in order to confirm the structure composition of "diversified" mechanism, distinguish the effect weight of each "unit" mechanism and offer more new valuable information for enhancing oil displacement process with steam injection.

\section{Exergy Transfer Paraphrase for Oil Displacement Process of Reservoir}

Exergy transfer is a basic law of studying exergy (quality of energy, available energy) transfer and conversion, and it is the cross boundary subject between thermodynamics and transmission science. Considering both dynamic and static character of energy, and also combined with quality and quantity of energy organically, therefore the true value of energy could be reflected and exergy transfer has high academic value and practical significance for optimizing transfer process and enhancing the energy consumption level. Through the analysis of evaluation standard of exergy flow density and change law of exergy transfer coefficient, the rationality of the process is evaluated and weak link is distinguished by exergy transfer analysis from dynamic point. At present, the exergy transfer study is roughly divided into two categories: one is focused on studying the principle and universal law of general exergy transfer phenomenon; the other is focus on studying the mechanism and calculation method of typical or specific engineering exergy transfer problems [5].

Exergy, a thermodynamics parameter, is defined as theoretical maximum work of physical distribution or system relative to environmental criteria. Analyzing the oil displacement process in reservoir from the energy 
conversion angle; it is the mass transfer process from an area of crude oil field to some production well at the cost of consuming some driving power. Driving power is one form of exergy (energy quality) transfer and conversion for potential field. Oil displacement process is a typical exergy transfer problem which takes the multi-potential field as driving source and oil displacement as purpose. Although a variety of potential fields corresponding to different types of exergy, all the exergy which has driving action reflects theoretical power relative to environmental criteria. Thus, unified as a single exergy effect and taking exergy calculation as a bridge, the synergistic effect of multi-potential field can be related to the final objective of some specific problem. Therefore, according to thermal driving process of complex mass transfer involving various energy forms at the same time, exergy transfer theory can change multiple objectives into a single target to study by leading in driving exergy, through which these problems can be simplified [6].

According to common target of various potential fieldsoffering driving exergy, united measure-contribution or effect of oil displacement process, numerous potential fields in driving domain are divided into four types: dynamic potential field, resistance potential field, destination potential field and ineffective potential field. Based on exergy transfer principle, the oil displacement mechanism can be summarized as following that, dynamic potential field offers directly power-driving work (exergy) to oil displacement and driving work is a unified criterion to evaluate the ability of any driving potential field; resistance potential field affects the oil displacement efficiency and effect in the way of weakening exergy transfer and producing exergy transfer resistance (exergy resistance); oil displacement process is a dynamic change process with the interaction of dynamic potential field and resistance potential field, and the integration of the dynamic characteristics of dynamic potential field and the resistance characteristics of resistance potential field determines the finial oil displacement effect. According to dynamics basic relationship of exergy transfer in oil displacement process and dynamics property of thermal driving synergy phenomenon, it indicates that the oil displacement process of multi-field synergy in steam injection is multi-level with the potential field as carrier, in which there are three levels among dynamic potential fields, among dynamic and resistance potential fields, and among resistance potential fields [7].

\section{The Contents of Urgent In-Depth Study}

\subsection{Exergy Balance Mathematical Model of Multi-Field Synergy for Oil Displacement Process in Steam Injection}

The essence of oil displacement with steam injection is exergy transfer process of multi-field synergy. Based on the previous research achievements, on the premise of each basic balance equations limiting system behavior and describing progressive law of process, replenishing the constitutive relation of calorifics-hydraulics-mechanics of oil reservoir, the exergy balance mathematical model with multi-field synergy for multiphase flow in porous media of steam injection reservoir is put forward considering the using of academic knowledge, such as irreversible thermodynamics, seepage mechanics, heat transfer, rock mechanics, etc. This model is closely interrelated with irreversible entropy production and also reflects the basic rules of spatial and temporal exergy distribution, which will lay foundation for in-depth analysis of oil displacement process with multi-field synergy in steam injection.

\subsection{Phenomenological Analysis of Exergy Transfer with Multi-Field Synergy for Oil Displacement Process in Steam Injection}

Based on bilinear form of different tensor rank of "force" and "flow" about entropy production rate that mainly caused by irreversible phenomena including seepage, heat transfer, mass transfer and the other phenomena of oil displacement process in steam injection, and through thermodynamics functions of general exergy flow and energy flow, the linear phenomenological equations of reflecting the relationship among power, resistance and rate in the multi-field synergy process of exergy transfer are built up. The conversion and transfer law of different exergy forms in oil displacement process with steam injection is discussed, the conversion and change laws of various exergy and the exergy loss mechanism in ireversible process are studied, especially determining the influence factors of various exergy transfer coefficient, thus the powerful basis for proposing controlling and regulating its corresponding exergy transfer process is provided.

\subsection{Oil Displacement Mechanism with Multi-Field Synergy in Steam Injection Based on Exergy Transfer Analysis}

From the perspective of general physics, seepage mechanics and irreversible thermodynamics, the types of multiphase flow in reservoir porous media caused by different driving potential fields are summarized comprehensively, and physical explanation of exergy transfer to oil displacement process of steam injection is studied deeply, the specific composition structure of dynamic potential field, resistance potential field and destination potential field in oil displacement region is attained. According to the study and determination of multi-field synergy evaluation index system of exergy transfer 
among dynamic potential fields, among dynamic and resistance potential fields, and among resistance potential fields in oil displacement process of steam injection, the oil displacement mechanism of multi-field synergy of how to enhance the production rate and oil reservoir producing degree meanwhile by improving dynamic characteristics and the resistance characteristics of driving exergy transfer process with steam injection is dissected at different levels.

\subsection{Experimental Study, Numerical Simulation and Application}

The analytical solution of the model in front is difficult to obtain generally; the typical steam driving process should be selected to analyze solution and application by numerical simulation method. Oil displacement mechanism of multi-field synergy in steam injection obtained by research should be certified and corrected through laboratory test.

\section{Adoptable Research Methods}

\subsection{Theoretical Model}

With field theory, the entropy equilibrium equation composed of entropy flow of outside supply and irreversible entropy production of system interior is deduced. According to the exergy general expression defined by generalized metric and extensive quantity of dead state thermodynamics, through satellite differential, the total exergy equilibrium equation is analyzed including such transfer forms as heat exergy, press exergy, move exergy and potential-energy exergy, etc. Based on irreversible thermodynamics, liner phenomenon-logical equations which reflect the relationship among dynamics, resistance and rate for exergy transfer process of multi-field synergy are built up. The composition structure of dynamics potential field, resistance potential field and destination potential field in oil displacement region is differentiated with seepage mechanics, general physics and irreversible thermodynamics. In the light of physical paraphrase of exergy transfer for oil displacement with steam injection, combined with the evaluation method of enhancing recovery in oilfield development region, the calculation index which reflects synergy relationship among dynamics potential fields, among dynamics and resistance potential fields, and among resistance potential fields of exergy transfer in oil displacement process is deduced, thus the exergy transfer analysis system of multi-field synergy with steam injection is set up.

\subsection{Numerical Simulation}

In accordance with the practical reservoir conditions, the fundamental mathematical model is built firstly, includ- ing the partial differential equation that describes fluid flow law in reservoir, the state equations and definite conditions that describe physico-chemical properties of fluid. Then the basic physical parameter of oil reservoir numerical simulation is confirmed through history matching of oil reservoir development performance. The evaluation index of exergy value, exergy transfer and multifield synergy for multiphase and multi-component mixture are calculated successively, and the basic exergy transfer law and oil displacement mechanism are studied after obtaining the numerical solution of the basic state parameters in oil displacement region. Finally, the influence of steam injection parameter on exergy transfer analysis is confirmed by numerical simulation, and then some suggestions and measures of improving oil displacement process can be proposed.

Numerical simulation uses the most common finite difference method, i.e., the continuity problems indicated by partial differential equations are dispersed with difference quotient instead of partial derivative, so the partial differential equations are dispersed into difference equations, and the corresponding algebraic equations are formed on finite points in solved region. The center network and five-point difference scheme can be used when dispersing the partial differential equations. The different equations are linearized with fully implicit or adopting implicit solution method for all variables. The LU decomposition method with D4 sorting or orthogonal minimization method of incomplete LU decomposition is selected to solve linearization equations. According to the temporal and spatial variation characteristics of related operating parameters in practical oil displacement process and combined with one part of necessary indoor experimental data, the operation details of grid direction and gird numerical quantity are determined reasonably.

\subsection{Experimental Verification}

The experimental means for directly measuring the calculation index of exergy transfer analysis, including exergy, exergy transfer coefficient and exergy flux density, etc. can not be provided at present. Seeing that exergy transfer analysis makes temperature, pressure and the other basic field parameters as calculation basis and its consequence is related to macroscopic criterions of oilfield development, such as recovery ratio, oil displacement rate and so on, the veracity and reliability of numerical simulation are verified directly or indirectly by quantitative contrast of the former and qualitative contrast of the latter. First of all, simulation crude oil is confected by the crude oil of steam driving test area. In accordance of the reservoir physical parameters of practical permeability and porosity in field, indoor physical displacement model is manufactured. The physical parameters of simulation oil and core including thermal conduc- 
tivity, density and specific heat capacity, etc can be determined by correlative instrument. The displacement experiment is conducted with the order of exhausting saturated water, saturated oil static, steam injection displacement. Temperature, pressure and the other basic physical parameters are taken notes, and then recovery ratio and oil displacement ratio are calculated from the test data, so that the direct and indirect verifications of numerical simulation result of exergy transfer are conducted respectively.

\section{Concluding Remarks}

To describe and analyze the displacement process of multi-field coupling with exergy transfer can simplify this kind of problem by introducing a unified goal-driving exergy. To study deeply the basic rule of exergy transfer in multi-field coupling flooding process, to make a thorough research for the flooding process of steam injection with exergy transfer theory, to reveal the oil displacement mechanism in three-dimensional heat region of multi-field coupling and to seek the cooperation mechanism which influence the common dependence and mutual restriction between dynamic potential field and resistance potential field, destination potential field and invalid potential field of exergy transfer in thermal oil displacement process, can merge the related theory of multi-subject, promote exergy transfer theory of the cross subject forward and enrich the research content of exergy transfer. Simultaneously, the obtained oil driving mechanism of multi-field coupling will provide theory instruction and technical support to make scientific development plan, enhance the reservoir mining effect and re- duce the mining cost for the heavy crude oil production enterprises.

\section{REFERENCES}

[1] Y. T. Zhang, “Technique for Improving Oil Recovery Efficiency with Thermal Recovery,” Petroleum Industry Press, Beijing, 2006.

[2] B. Zou, P. Y. Gai and W. F. Song , "Review on Steam Flooding and Study Trend in Shengli Oilfield,” Petroleum Geology and Recovery Efficiency, Vol. 17, No. 5, 2010, pp. 50-52.

[3] Y. L. He and Z. Q. Xu, "Parameter Optimization on Steam Drives for Wa 38 Fault Block Pilot, Xiaowa Oilfield,” Petroleum Geology and Recovery Efficiency, Vol. 17, No. 3, 2010, pp. 77-79.

[4] S. L. Zhuang and Y. X. Luo, "Experimental Study for Enhancing Oil Recovery with Transfer from WaterDriving to Steam-Driving in Fuyu Oilfield," Journal of Yangtze University, Vol. 6, No. 2, 2009, pp. 38-41.

[5] Y. Liu, Q. L. Cheng and X. Y. Xiang, "Driving Process of Multi-Field Synergy with Exergy Transfer Analysis," Journal of Daqing Petroleum Institute, Vol. 34, No. 5, 2010, pp. 53-59.

[6] Q. L. Cheng, Y. Liu and X. Y. Xiang, "The Physical Foundation for Describing Reservoir Oil Displacement Process with Exergy Transfer," Journal of North China Electric Power University, Vol. 37, No. 1, 2010, pp. 1013.

[7] Q. L. Cheng, Y. Liu and Z. G. Wang, "The Description and Analysis of Multi-Field Synergy for Thermal Driving Process of Heavy Oil," Journal of Engineering Thermophysics, Vol. 31, No. 5, 2010, pp. 737-741. 